\title{
Further Remarks on Extra Roots of Rayleigh Equation and Somigliana Waves
}

\author{
A. L. LevsinN $(*)-G$. F. PANZA (**) \\ Ricevuto il 24 Giugno 1970
}

\begin{abstract}
Riassunto. - Le radici magrgiori dell'unitì dell'equazione di Rayleigh applicate ad un semispazio elastico contribuiscono alla soluzione solo per valori abbastanza grandi del coefficiente di Poisson $(\sigma>0.309)$. Una delle radici corrisponde ai "leaking modes" i quali hanno velociti di fase minore della velocità delle onde longitudinali. Un'analoga onda con diversa dispersione può esistere nel caso in cui un semispazio elastico è coperto da uno strato caratterizzato da velocitì $\alpha$ e $\beta$ minori di quelle del semispazio. Lo spessore dello strato non deve essere troppo piccolo in corrispondenzal della lunghezza d'onda.
\end{abstract}

Sumarr. - The extra roots of the Rayleigh equation for an elastic halfspace contribute to the solution only for large enough values of the Poisson coefficient $(\sigma>0.309)$. One of them corresponds to leaking modes with the phase velocity less than the velocity of the longitudinal wave. $\Lambda$ similar wave with distinct dispersion may exists in the case where an elastic halfspace is covered by a thin layer with lower velocities of elastic waves. The thickness of a layer should be not too small in comparison with the wave length.

\section{INTRODUCTION.}

Since the basic paper of Rayleigh ( $\left.{ }^{1}\right)$ on surface waves in an elastic halfspace was published, a significant number of papers have considered the physical meaning of extra roots of the Rayleigh equation.

(*) Soviet Geophysical Committee, Moseow (USSR).

(**) Istituto di Fisica, Istituto di Creodesia, Bologna (Italia). 
Recently we have the papers of Caloi $\left({ }^{2}\right),\left({ }^{3}\right),\left({ }^{4}\right)$, Caloi and Romualdi $\left({ }^{5}\right)$ which link at least one of these roots to the Somigliana waves and give some physical arguments and experimental data for their existence.

In this review we want to reconsider this problem, basing our arguments on some results of Ewing, Jardetzky and Press $\left({ }^{6}\right)$. Phimney (7), Gilbert and Isaster $\left({ }^{8}\right)$, Gilbert $\left({ }^{9}\right)$.

\section{1. - Plane rares in a halfspace.}

Let us consider an elastic homogeneous halfspace and let $\alpha$ and $\beta$ be the velocities of $P$ and $s$ waves $\gamma=\left(\frac{\beta}{\alpha}\right)^{2}$ and $\sigma$ be the Poisson's ratio.

The fundamental Rayleigh equation for unknown phase velocity $c$ of surface wave rumning along a free surface is

$$
F(c)=\left[2-\left.\left(\frac{\tilde{\alpha}}{\hat{p}}\right)\right|^{2}-4 \mid 1-\left(\frac{c}{\hat{p}}\right)^{2}\right]^{1 / 2}\left[1-\left(\frac{c}{\beta}\right)^{2} \gamma\right]^{1 / 2}=0 .
$$

Like Rayleigh we assume that the signs of radicals are positive, then the equation has only one positive real root $c_{1}\left(0<c_{1}<\beta\right)$, which is of course the Rayleigh root.

If the signs of radicals are not specified two additional (extra) roots arise $\left(c_{2}\right.$ and $\left.c_{3}\right)$; if $\sigma>\sigma_{c}\left(\sigma_{c}=0.263082\right)$ both roots are complex and conjugated; moreover if $\sigma>\sigma_{s}\left(\sigma_{s}=0.309\right)$ then the real part of $e_{2}$ and $c_{3}$ is less than $\alpha$, i.e. with usual notations $R e e_{2,3}<\alpha$; finally if $\sigma_{c}<\sigma<\sigma_{s}$ then $R e c_{2,3}>\alpha$.

For $\sigma<\sigma_{c}$ both roots are real and $c_{3}>c_{2}>a$. These real roots are linked to waves which Caloi called Somigliana waves. Fig. 1 gives the roots versus $\sigma$.

It was shown by Fu ( $\left.{ }^{10}\right)$ that the phase velocities $c_{2}$ and $c_{3}$ correspond to such angles of emergence $e_{2}$ and $e_{3}$ of the plane waves at the free boundary for which reflected plane waves of the same kind do not exist; this is expressed by the following formulae

$$
\begin{array}{lll}
\operatorname{tg} e_{2}^{P}=\sqrt{\frac{c^{2}}{\alpha^{2}}-1} & ; & \operatorname{tg} e_{3}^{P}=\sqrt{\frac{e^{2}{ }^{2}}{\alpha^{2}}-1} \\
\operatorname{tg} e_{2}^{S}=\sqrt{\frac{c^{2} 2}{\beta^{2}}-1} & ; & \operatorname{tg} e_{3}^{S}=\sqrt{\frac{c^{2} \beta^{2}}{\beta^{2}}-1} .
\end{array}
$$




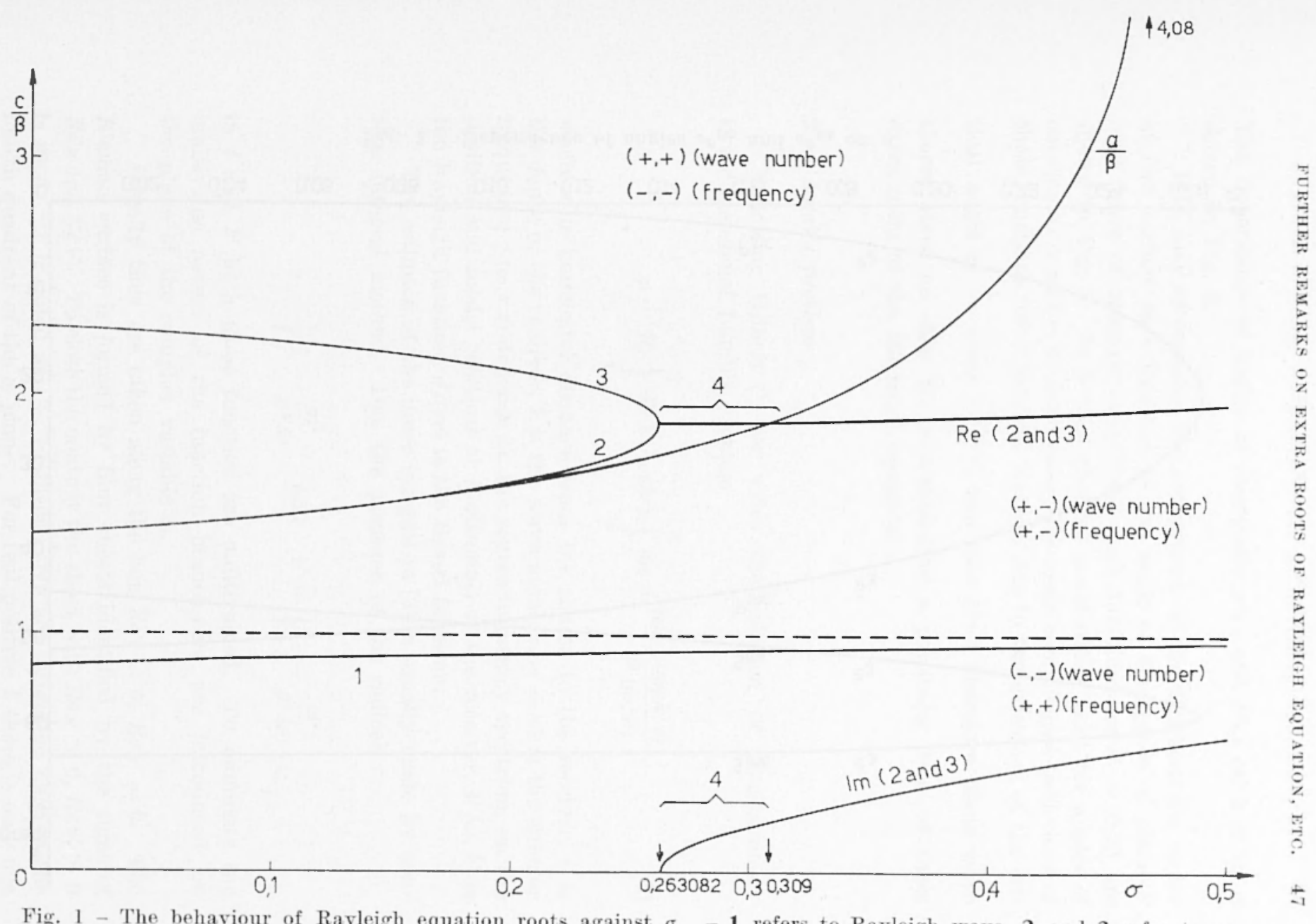

Fig. 1 - The behaviour of Rayleigh equation roots against $\sigma$. -1 refers to Rayleigh wave. 2 and 3 refer to so ealled Somigliana waves. Re $(2$ and 3$)$ refer to $P L$ waves. 4 refers to no accessible $P L$ modes. Arrows indicate the eritical values of $\sigma$. Plus and minus in brakes indicate the sign of radicals, i.e. the Rieman sheets. 


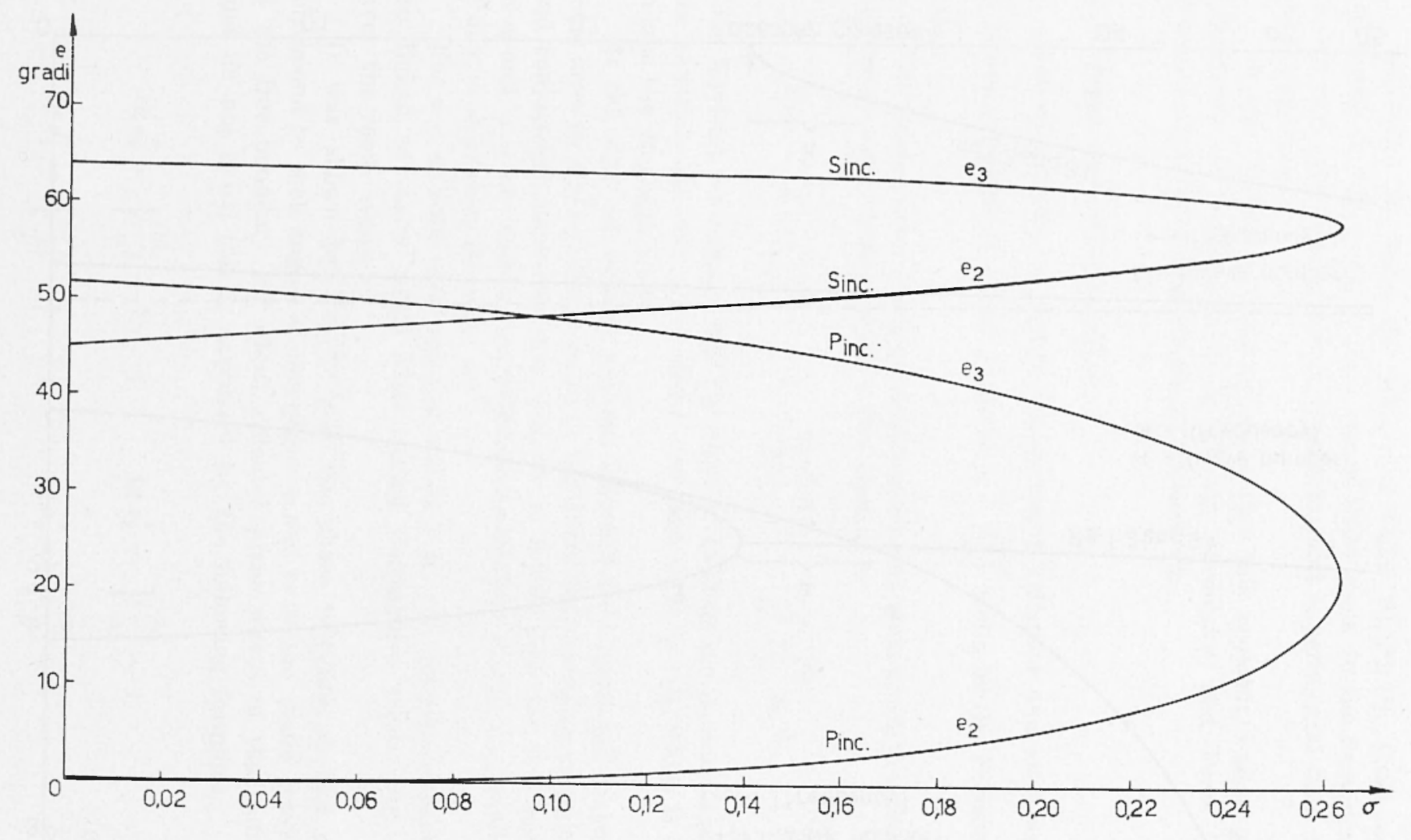

Fig. 2 - Dependence of angles $e^{\bar{r}}{ }_{2,2}$ and $e^{S_{2,3}}$ on $\sigma$. 
The dependence of angles of emergence $e^{P_{2,3}}$ and $e^{S_{2,3}}$ on $\sigma<\sigma_{c}$ is shown in Fig. 2.

It is easy to compute the components of the displacement vector at free surface as a function of the angle of emergence of plane $P$ or $S$ wave of constant amplitude. Such functions for $\sigma=0,25$ are shown in Fig. 3. It is seen that no peculiarities exist for angles of emergence $e_{2}$ and $e_{3}$ in both cases; the more complicated behaviour of these functions for $S$ incident wave is due to the existence of the critical angle $e_{c}=\operatorname{arcos}\left|\frac{\beta}{\alpha}\right|$ in this case $\left({ }^{11}\right)$. Therefore plane wave theory gives no clew for understanding a particular role of these extra roots of the Rayleigl equation.

\section{2. - Lamb's problem.}

Following Gilbert $\left({ }^{9}\right)$ we write the solution of nonstationary two-dimensional Lamb's problem

$$
p=\left.R_{e} \int_{0}^{\infty} d k k J_{o}(k r)\right|_{0} ^{\infty} d(1) e^{-i \omega v} \frac{f(\omega, k, z)}{F(\omega, k)}
$$

where $r$ is horizontal distance from the source to the receiver, $z$ is the depth of the receiver, $k$ is the wave number, $\omega=c k$ is the circular frequency; $f(\omega, 7, z)$ depends on the source frequency spectrum, on the position and model (vertical or horizontal) of the receiver. $F(\omega, k)$ is the Rayleigh function, $J_{o}(k r)$ is the Bessel function.

The estimate of the inner integral in [3] is usually made by contour integral method. Due the presence of the radicals

$$
v=\sqrt{1-\frac{\omega^{2}}{\alpha^{2} k^{2}}} \text { and } v^{\prime}=\sqrt{1-\frac{\omega^{2}}{\beta^{2} k^{2}}}
$$

in $f$ and $F$ both these function are multivalued. To eliminate the multivalue nature of the function branch-cuts are introduced on the plane of the complex variable $\omega$.

Usually they are taken along the line $R e v=0, R e v^{\prime}=0$. The Rieman surface is formed by four sheets identified by the signs of $\operatorname{Re} \nu$ and $\operatorname{Re} \boldsymbol{v}^{\prime}$. To close the contour the sheet with $\operatorname{Re} v>0, \operatorname{Re} \boldsymbol{v}^{\prime}>0$ is used. It is noted as $(+,+)$ sheet. We are interested only in the fourth quadrant of the $\omega$ plane. For real positive $k$ there is only one 


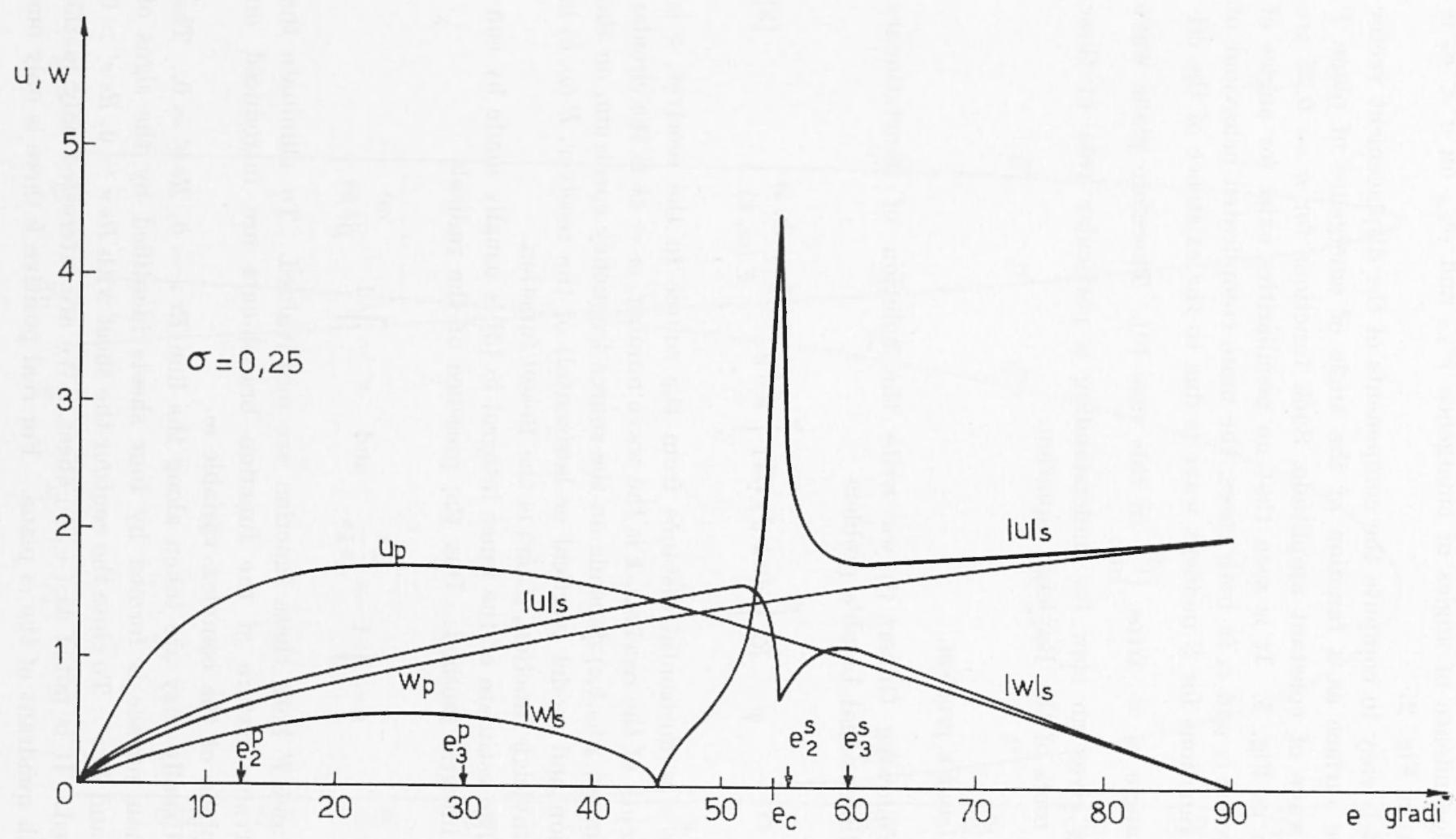

Fig. 3 - Components of the displacement vector of free surface as a function of the angle of emergence $e$. 
simple pole of the integrand related to the Rayleigh root of the equation $F(\omega$, li $)=0$ namely $\omega=k c_{1}$.

The contribution of the body waves is described by the integrial along the branch lines (Fig. 4a).

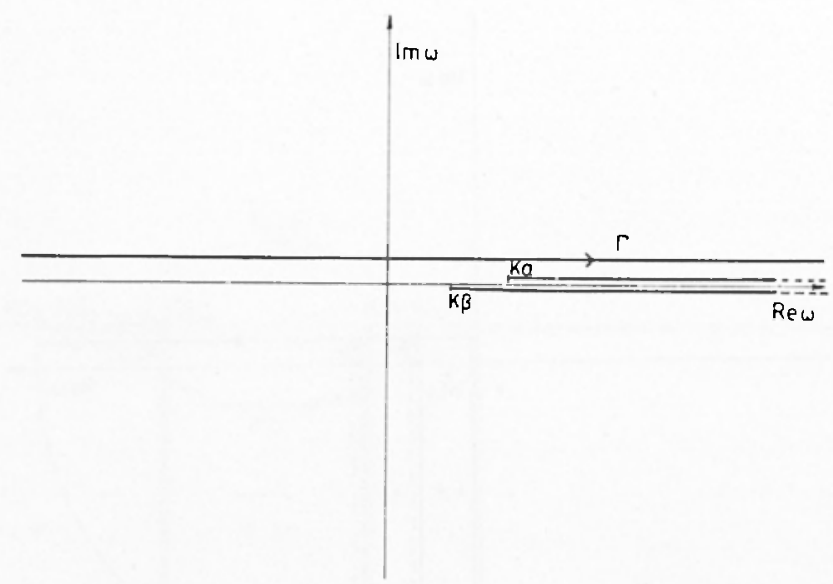

Fig. 4a - Branch points, branch cuts, initial contour of integration on complex plane.

This method of describing the contribution of the body waves is of course not unique. If we cut the branches vertically down from branch points $\omega=k \alpha, \omega-k \beta$ and we keep the upper part of the plane on the same sheet as before, it is possible to uncover previously inaccessible parts of other sheets with $R e v>0, \operatorname{Re} v^{\prime}<0(+,-)$ and $\operatorname{Re} \nu<0, \operatorname{Re} \nu^{\prime}<0(-,-)$.

Then our solution may be presented as a contribution to the integrals along the new branch cuts describing main body waves (because of the $k_{\alpha}$ and $k_{\beta}$ poles) plus the contribution of the Rayleigh pole plus the contribution of the poles from the uncovered parts of the $(+,-)$ and the $(-,-)$ sheets if such poles exist (Fig. 4b).

At our case the poles at the uncovered part of $(-,-)$ sheet are absent. For $\sigma<\sigma_{c}$ the poles $\omega=k c_{2}$ and $\omega=k c_{3}$ related to the extra roots of [1] are situated to the right of $\omega=k \alpha$ on inacessible part of $(+,-)$ sheet and do not contribute to our solution. The same situation exists for complex poles $R e \omega_{2,3} \pm i \operatorname{Im} \omega_{2,3}$ when $\sigma<0,309$;

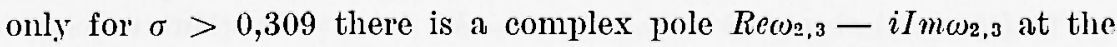
accessible part of $(+,-)$ sheet which contributes to the solution as so called $\bar{P}$ pulse or $P L$ wave (e.g. Phinney $\left({ }^{5}\right)$, Gilbert and Laster $\left({ }^{8}\right)$, 
Nanuchov, Agurtsoff $\left({ }^{13}\right)$. This wave is a weak disturbance behind $P$ wave. It becomes more and more distinct as $\sigma$ increases.

As extra roots do not contribute to the solution for $\sigma<0.309$, Somigliana waves do not exist in this case.

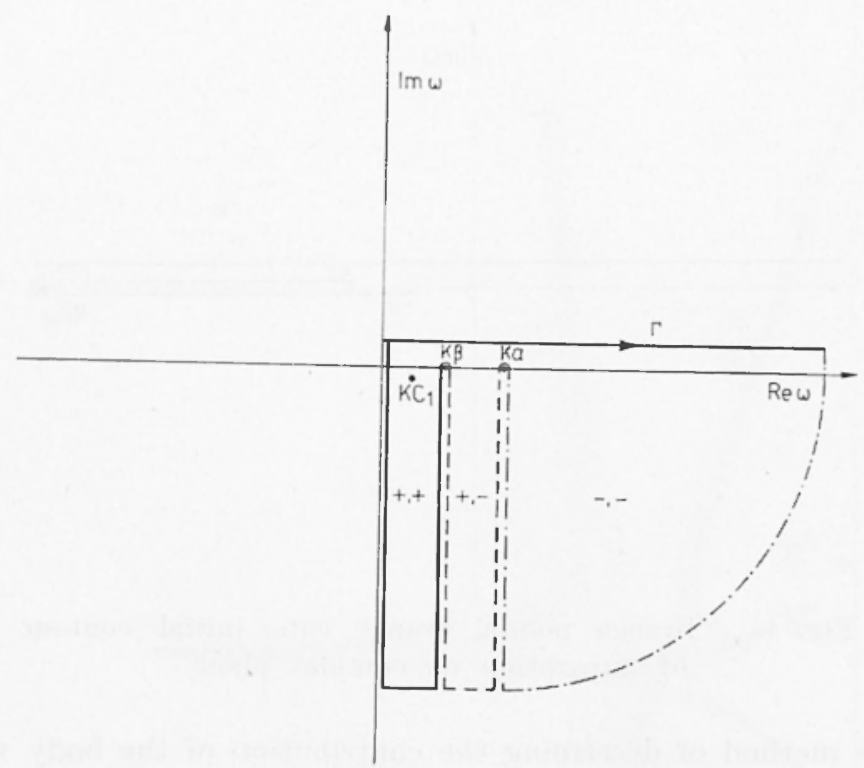

Fig. 4b - Branch points, branch cuts, used contour of integration on complex plane.

\section{3. - Layered halfspace.}

Let us consider the effect of thin elastic layer overlaying the same elastic halfspace with $\sigma<\sigma c$. Such a layer as Caloi (') mentioned is necessary to generate the Somigliana waves. The thickness of a layer is $H$ and its compressional and shear velocities are $\alpha_{0}$ and $\beta_{0}$ respectively $\left(\beta_{o}<\beta\right)$. The formula of displacement looks like [3] but the function $f$ and $F$ should be replaced by the new functions $F^{*}(\omega, k, z)$ and $F^{*}(\omega, k)$ which tend to $f$ and $F$ as $H \longrightarrow 0$.

Let us fix $k>0$. Then for infinitely small $k H$ we have the same situation as before. As $H$ increases the poles of $F^{*}(\omega, k)$ shift from their positions for $H=0$. The Rayleigh pole keeps on the accessible part of $(+,+)$ sheet moving from $\omega=k c_{1}$ to $\omega=k c_{o}$ (here $c_{o}$ is velocity of Rayleigh wave at the halfspace with velocities of body waves $\alpha_{0}$ and $\beta_{n}$ along the real axis). The behaviour of other poles is much 
more complicated. The couple of poles on the real axis to the right of $\omega=l i \alpha$ situated on the covered part of $(+,-)$ sheet unites in the conjugated couple and moves to the left as $H$ increases. The pole which moves at the fourth quadrant eventually crosses the $P$ branch-cut

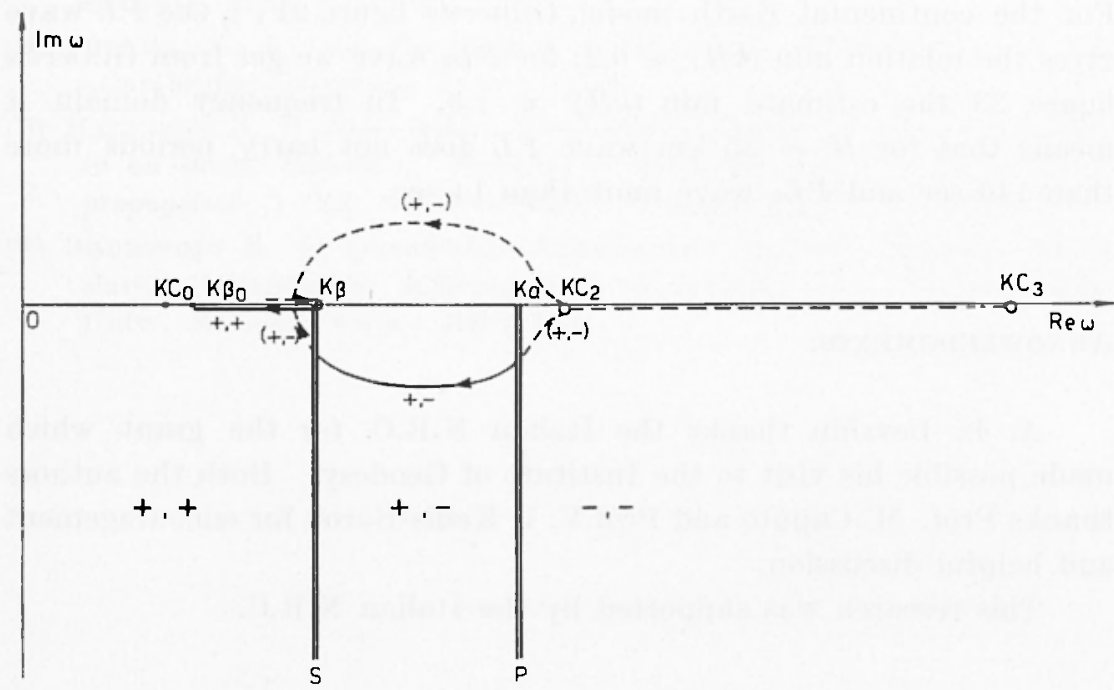

Figr. 5 - Movements of pole $P_{+}$for fixed $k$ and variable $I I$.

to appear on the open part of $(+,-)$ sheet. Only now it begins to contribute to the solution as dispersive leaking mode $P L\left(\bar{P}_{+-}\right)$. As $H$ increases more it crosses the uncovered stripe of the $(+,-)$ sheet and $S$ branch-cut, to the covered part of the same sheet. Here it unites to the conjugated pole at the second quadrant to become real. Then one of them still keeps on the same part of $(+,-)$ sheet, and the other one overtakes the branch-cut to appear on accessible part of $(+,+)$ sheet to the left of $(1)=k \beta$. Now it contributes as the first shear mode and its evolution is limited by points (I) $=k \beta_{o}$ and $(1)=k \beta$ (Fig. 5).

In a similar way the other couple of real poles on the covered $(-,-)$ sheet unites at the conjugated couple as $H$ increases. The pole at the fourth quadrant after some evolutions appears on the uncovered part of $(+,-)$ sheet to contribute as leaking mode $P L_{3}\left(P_{-+}\right.$ of Gilbert). Then if $H$ is large enough it goes to $(+,+)$ sheet to become the third shear mode. 
We do not discuss here the behaviour of other poles of $F(\omega, k)$ which are not related to the roots of Rayleigh equation.

Thus the existence of the surface layer really "wakes up" the extra roots of Rayleigh equation permitting them to contribute to the solution. However this happens only if the layer is thick enough. For the continental Earth model, Gilbert's figure $21\left(^{\circ}\right)$, the $P L$ wave grives the relation min $(k H) \approx 0.2$ : for $P L$, wave we get from Gilbert's figure 23 the estimate $\min (k H) \approx 1.9$. In frequency domain it means that for $H=3 \check{5} \mathrm{~km}$ wave $P L$ does not carry periods more than 140 sec and $P L_{3}$ wave more than $14 \mathrm{sec}$.

\section{AKNOWLEDGMENTs.}

A. I. Levshin thanks the Italian N.R.C. for the grant which made possible his visit to the Institute of Geodesy. Both the authors thanks Prof. M. Caputo and Prof.V. I. Keilis-Borok for encouragement and helpful discussion.

This research was supported by the Italian N.R.C.

\section{REFERENCES}

(') Rayleigh L., On Waves Propagated along the Plane Surface of an Elastic Solid. "Proc. Tondon Math. Soc." 17, 4-11, 1885; or "Sci. Pajers ", 2, 441.447; "Cambridge Univ. Press", London (1900).

(-) Canor P., L'equazione di Rayleigh e le onde di Somigliana. II. La teoria di Somigliana rellifiche e conseguenze. "Atti Acc. Naz. Lincei, Classe Scienze Fis. Mat. e Nat.", XLI. 5 (1966).

$\left({ }^{3}\right)$ Canor P., On the Upper Manlle. "Allvances in Geophysies", 79-211. "Academic Press", New York and London (1967).

(') Calor P., L'equazione di Rayleigh e le onde di Somigliana. Loro imporlanza per lo studio della crosta terrestre. "Atti Acc. Naz. Lincei. Classe Scienze Fis. Mat. e Nat., "XLIII, 6 (1967).

(5) Calor P. and Romuador G., More on the Somigliana Waves. "Annali di Geofisica ” XXI, 3, 239-264 (1968).

$\left(^{6}\right)$ Ewing W. M., Jandetzky W.S. and Press F., Elastic Waves in Layered Media. "Me Graw Hill Book Company, Inc." New York, Toronto and London (1957).

(7) Puynney R. A., Propagation of Teaking Inlerface Waves. "Bull. Seism. Soc. Ant. n, 51, 9 (1961). 
PCR'TIER REMARKS OS EXTRA ROO'TS OF RAYLEIGH EQUATION, E'TC. 5j

(8) Gilbert F. and I.aster, J. I., Exilation and Propagalion of pulse on an Interface. "B.S.S.A.", 52, 299.319 (1962).

(') Gilbert F., Propagation of transient leaking modes in a stratifted elastic wave guide. "Rev. Geophysies", 2, 123-153 (1964).

(19) Fu C. Y., On the irrelevant roots of the Rayleigh Wave Equation. "Science Record ", 2, N. Y. (1949).

(11) Buldes, K. E., An Introduction to the Theory of Seismology. p. 106, "Cambridge University Press"(1963).

(12) Manucirov A. Y., Some exact theoretical seismograms for wave propagation in an elastic haljspace. Problems of dynamical theory of seismic wate propagation, 1 (Ed. G.I. Petrashen), Leningrad [1957 (in Russian)].

(13) OGurtsoff K. I., Quantitative Investigations of Ware Dynamics in an elastic IIalyspace for different kinds of sources. "Proc. Ieniugradsky Univ.. Mathem. Seria " 208 (1956). 